\title{
Wideband PowerLine Positioning for Indoor Localization
}

\author{
Erich P. Stuntebeck \\ GVU Center \\ School of Interactive \\ Computing \\ Georgia Institute of \\ Technology \\ eps@gatech.edu
}

\author{
Shwetak N. Patel \\ DUB Group \\ Computer Science and \\ Engineering, Electrical \\ Engineering \\ University of Washington \\ shwetak@cs.washington.edu
}

\author{
Thomas Robertson \\ GVU Center \\ School of Interactive \\ Computing \\ Georgia Institute of \\ Technology \\ troomb1@cc.gatech.edu
}

\author{
Matthew S. Reynolds \\ Department of Electrical \\ and Computer Engineering \\ Duke University \\ matt.reynolds@duke.edu
}

\author{
Gregory D. Abowd \\ GVU Center \\ School of Interactive \\ Computing \\ Georgia Institute of \\ Technology \\ abowd@cc.gatech.edu
}

\begin{abstract}
Fingerprinting techniques for indoor localization have been widely explored. A particular approach by Patel et al. suggested leveraging of the residential powerline as the signaling mechanism for a domestic location capability. In this paper, we critically examine that initial work, called powerline positioning (PLP). We find the proposed technique lacking in temporal stability, requiring frequent and undesired recalibration in some environments. We also determine that there is no a priori method to determine a pair of signaling frequencies that will reliably work in any space. We propose a wideband approach to PLP (WPLP) that injects up to 44 different frequencies into the powerline. We show that this WPLP approach improves upon overall positioning accuracy, demonstrates greatly improved temporal stability and has the added advantage of working in commercial indoor spaces.
\end{abstract}

\section{ACM Classification Keywords}

H.4.m Information Systems Applications: Miscellaneous

\section{General Terms}

Performance, Design, Reliability, Experimentation

\section{Author Keywords}

Location tracking, Localization, Context awareness, Fingerprinting

\footnotetext{
Permission to make digital or hard copies of all or part of this work for personal or classroom use is granted without fee provided that copies are not made or distributed for profit or commercial advantage and that copies bear this notice and the full citation on the first page. To copy otherwise, or republish, to post on servers or to redistribute to lists, requires prior specific permission and/or a fee.

UbiComp'08, September 21-24, 2008, Seoul, Korea.

Copyright 2008 ACM 978-1-60558-136-1/08/09...\$5.00.
}

\section{INTRODUCTION}

Ever since the seminal work on RADAR [4], many examples of RF fingerprinting have shown how existing infrastructure for mobile communications, primarily WiFi and GSM, can be leveraged to provide location information. Patel et al. discussed some of the downsides of relying on wireless access points and cell towers for a domestic indoor location system and proposed the alternative use of residential power lines [16]. With powerline positioning (PLP), the electrical infrastructure acts as an antenna, and artificially-generated signals transmitted over this antenna demonstrate the spatially differentiable and temporally stable signal map that is necessary for a fingerprinting approach to work.

In this paper, we critically examine some of the important details of the Patel et al. PLP proposal, presenting evidence that the initial approach suffers from temporal instability in certain environments. The original PLP solution suggested the use of a pair of frequencies independently injected into the power lines at separate points in the house. Though the initial results were promising, we show that it is not possible to select a specific pair of frequencies that can be guaranteed to work in every setting over a period of time even as short as two months. If a solution to this problem cannot be found, frequent labor-intensive retuning and recalibration of the entire system is needed. This will severly limit this system's acceptance in commercial and home environments.

This is not only a concern of PLP, but of all fingerprintingbased localization systems. To date, most fingerprintingbased indoor localization work has been carried out with evaluation datasets obtained within a short time (hours to a few days) of the training data set. In this work we consider the implications of time separating the training and evaluation data sets and show that the temporal stability of a naïve fingerprinting approach can be much worse than expected, depending on the variability of signal generation and propagation. We then show that the use of observations across multiple frequencies, which we call Wideband PowerLine 
Positioning (WPLP) can help mitigate the effect of temporal variation in signal propagation.

\section{RELATED WORK}

Indoor positioning has been a very active area of research in ubiquitous computing for the past decade [9] and a variety of commercial systems are beginning to emerge. Some of the first indoor solutions introduced new infrastructure to support localization $[22,7,8,17,18,19,21]$. Despite some success, as indicated by commercialized products $[5,2,20$, 3 ], the cost and effort of installation are a major drawback to wide-scale deployment, particularly in domestic settings. Thus, new projects in location-based systems research reuse existing infrastructure to ease the burden of deployment and lower the cost.

The earliest demonstrations leveraged WiFi access points $[4,13]$, and shortly afterwards researchers began to explore Bluetooth [14] and wireless telephony infrastructure, such as GSM $[6,12,15]$ or FM transmission towers [11]. A concern is that individuals may not be able to control the characteristics of this infrastructure and the operational parameters of the infrastructure may change without warning, resulting in the need to recalibrate, such as in the case of GSM and WiFi localization. Individuals may opt to install their own WiFi infrastructure in their home. However, the number of base stations required for effective localization may be undesirable, because of the overhead of installing many units.

The desire to control the infrastructure and to scale inexpensively to track a large number of objects inspired Patel et $a l$. to work on a positioning system that leveraged the powerline infrastructure in a home [16]. The system requires only two transmitter modules to be installed in the home and compares favorably to GSM and WiFi-based localization systems. The appeal of this approach inspired us to provide a deeper understanding of the powerline-based localization approach and address the limitations of the prior work, which will be described shortly, by presenting a new wideband technique.

\section{Localization Techniques}

Traditional wireless signal triangulation, such as WiFi access point triangulation, uses Received Signal Strength Indicator (RSSI) information to estimate distance and determine a location based on its geometric calculations. Other techniques include the use of Time of Arrival, as in the case of ultrasound, or Angle of Arrival, such as with Ultra-wideband positioning [20]. Ultrasonic solutions, such as Cricket [17] and Active Bat [22], provide precise centimeter resolution, but require line-of-sight operation indoors. Therefore, they require dense sensor installations for full coverage. Technologies that avoid issues of occlusion, such as WiFi triangulation, suffer from multipath problems caused by reflections in the environment. Thus, fingerprinting of the received signals can help overcome the multipath problem by leveraging the multipath phenomenon [10]. Fingerprinting improves on other means of estimation by taking into account the effects that buildings, solid objects, or people may have on a wireless or RF signal, such as reflection and attenuation. Finger-

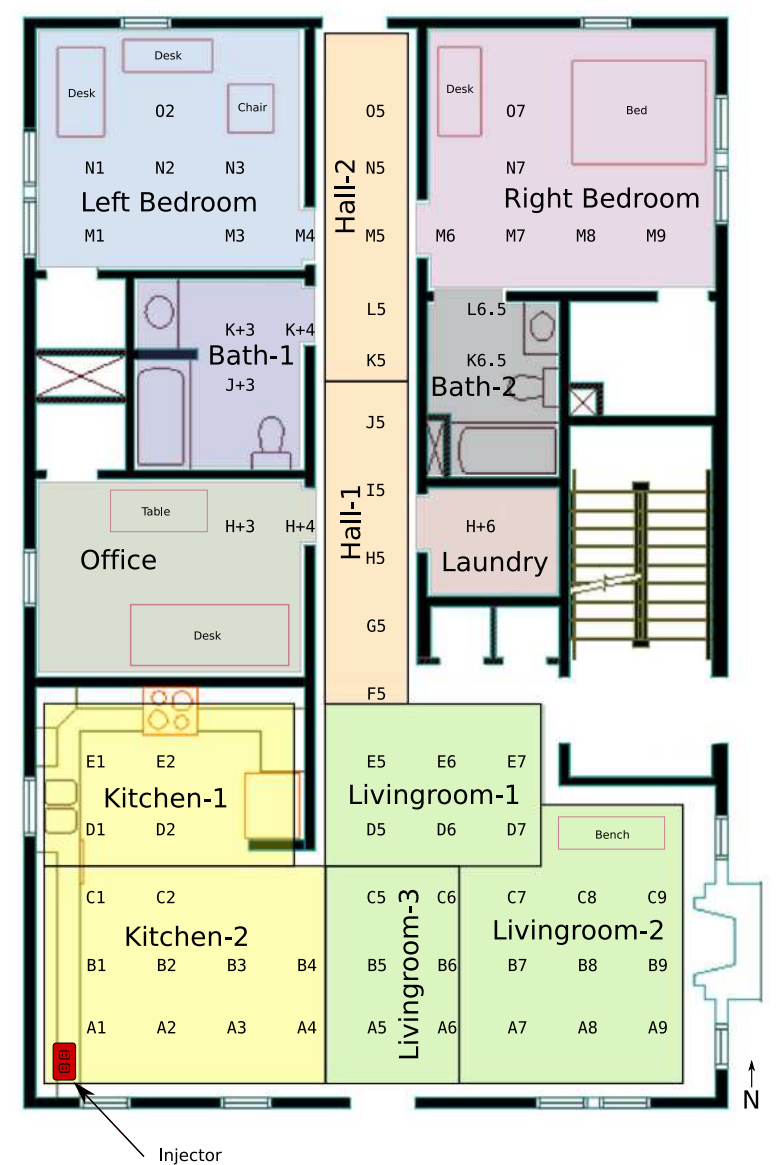

Figure 1. Floorplan and associated room-level, sub-room-level, and grid-level labels of the space where tests were conducted. Only labeled grid-points were surveyed. The signal injector was connected to an outlet in the kitchen as marked. Note that it is not necessary to know the layout of the in-wall electrical wiring. The only purpose of the wiring is to radiate the signal used for fingerprinting throughout the space of interest.

printing works by recording the characteristics of wireless signals at a given position and later inferring that position when a similar signature is seen again. A survey of signals over some space allows for the creation of a map that can be used to relate a signal fingerprint to a location.

\section{EXPERIMENTAL SETUP}

We conducted experiments using our prototype system in a research laboratory designed to imitate a residential environment. The building contains two floors with identical floorplans, each with a kitchen, livingroom, two bedrooms, two bathrooms, and an office. Although the facility appears to be a home, it differs in two important ways from a typical home. First, the facility contains a large number of computers and other electronic devices, leading to a noisier RF environment than true residential spaces. Second, the facility was constructed to meet industrial building codes, which require electrical wiring to be surrounded by metal conduit. This metal conduit, which can block the signals injected over the powerlines from radiating as RF energy, is neither required nor common in most residential construction. These fac- 

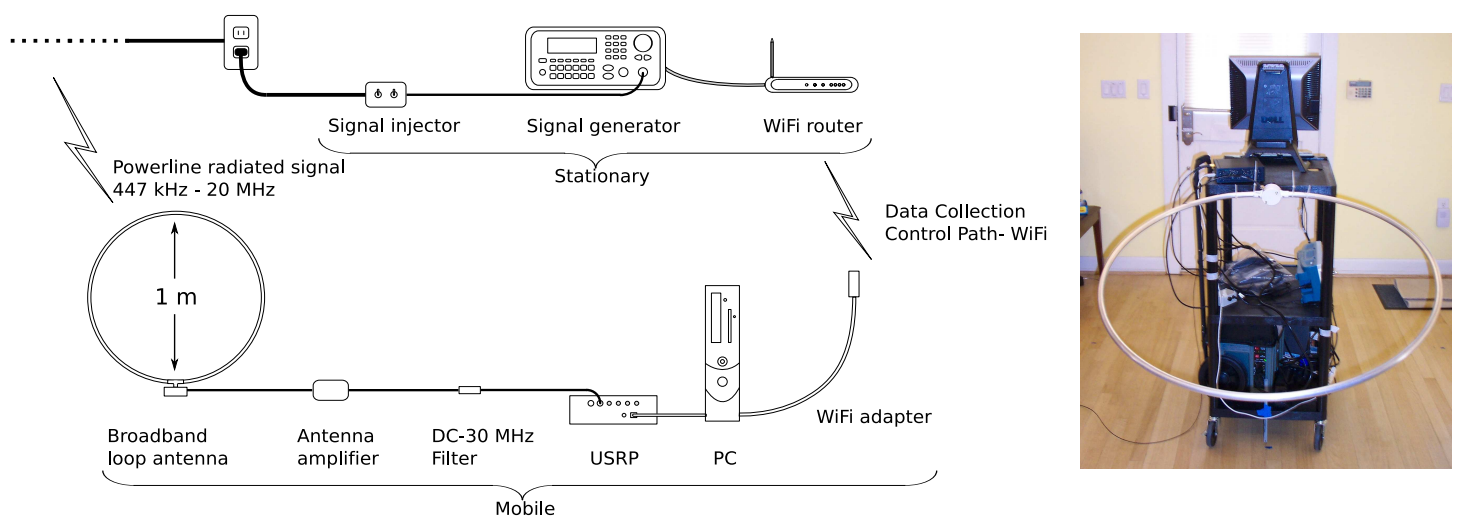

Figure 2. Equipment used to transmit and collect the amplitude of the wideband signal. The WiFi connection is used only to remotely control the signal generator, and is not in any way used for localization.

tors mean that the performance of our system would likely improve in a true residential environment, and that our results with WPLP would extend to other commercial indoor spaces.

\section{Grid Layout}

Survey points consisted of 66 locations throughout the first floor on a 0.9 by 0.9 meter grid, shown in Fig. 1. Each point on the grid was identified by a letter (A-O) for the row and a number (1-9) for the column. Occasionally, due to the layout of the floor, surveyable points fell halfway between major grid points. These points are designated by a '.5' in the column number for a point falling halfway between two columns, and a ' + ' after a row letter for a point falling halfway between two rows. Due to the size of the antenna ( $1 \mathrm{~m}$ diameter) relative to the size of the grid, its orientation at each point can be a significant factor. Data was obtained at each of the 66 points with the antenna facing either north, south, east, or west. One direction was used for each point, and the same direction was used each time data was taken at that point. Most points were sampled with the antenna facing north, although occasionally the placement of walls and furniture dictated a different orientation.

\section{Data Collection Equipment}

To generate the signal that was injected into the powerlines, we used an Agilent 33220A $20 \mathrm{MHz}$ signal generator to produce a $10 \mathrm{~V}$ peak-to-peak sine wave. Forty-four different frequencies were used in total $-447 \mathrm{kHz}, 448 \mathrm{kHz}, 600$ $\mathrm{kHz}$, and $601 \mathrm{kHz}$ (Patel et al. PLP-I frequencies), and 500 $\mathrm{kHz}$ to $20 \mathrm{MHz}$ in $500 \mathrm{kHz}$ steps. We were limited to 20 $\mathrm{MHz}$ and $10 V_{p p}$ by the capabilities of the signal generator. Also, it is important to note that the signal generator expects a $50 \Omega$ load, and will only properly generate a 10 $V_{p p}$ sine wave when matched with such a load. Our experiments have shown that the impedance of the powerline varies by frequency, and is usually much higher than $50 \Omega$. Therefore, the true voltage injected into the powerlines varied between $2 V_{p p}$ and $9 V_{p p}$ depending on the frequency. Although frequencies at which the powerline's impedance closely matches that of the signal generator will radiate more energy, this will not affect our results as long as the power- line impedance is relatively consistent. This should be the case as long as there are no significant changes in the electrical infrastructure of the space during the experiments.

The output of the signal generator was connected to a custombuilt powerline injector box which gave us the capability to inject the signal on the hot and neutral, hot and ground, or neutral and ground wires. To compare our results as closely as possible with those of Patel et al. and PLP-I, we chose to inject the signal on the hot and neutral wires. However, work in progress suggests that using the metal conduit itself to distribute the signal may prove more effective in industrial environments.

The receiver consisted of an amplified broadband antenna, a software radio, and a standard laptop computer. The antenna, a Wellbrook Communications ALA-1530+ loop antenna, had a range of $150 \mathrm{kHz}-30 \mathrm{MHz}$ [1]. The software radio, an Ettus Research Universal Software Radio Peripheral (USRP), contained a $64 \mathrm{MHz}$ 12-bit analog-to-digital converter to which the output of the antenna was connected through a low-pass antialiasing filter. A large battery and a power inverter were used to power the equipment in order to both isolate it from the powerline and to make it electrically similar to a battery-powered mobile tag.

All components of the receiver (laptop, antenna, and software radio) were placed onto an electrically isolated plastic cart to ensure no signal was coupled through the cart. The signal generator and injector box were placed in the kitchen, and remained stationary throughout the experiments. The equipment setup is shown in Fig. 2.

Although we realize that the size and complexity of this experimental setup makes it impractical for a real-world deployment, we nonetheless decided on the use of a broadband antenna and software radio to allow rapid prototyping of a variety of ideas. Our prototype has much more functionality than necessary for a real-world system and can easily be reduced in complexity. The design of a real-world deployable prototype is not the contribution of this paper, however we emphasize that it is possible and later describe how a deployable version might be built. 


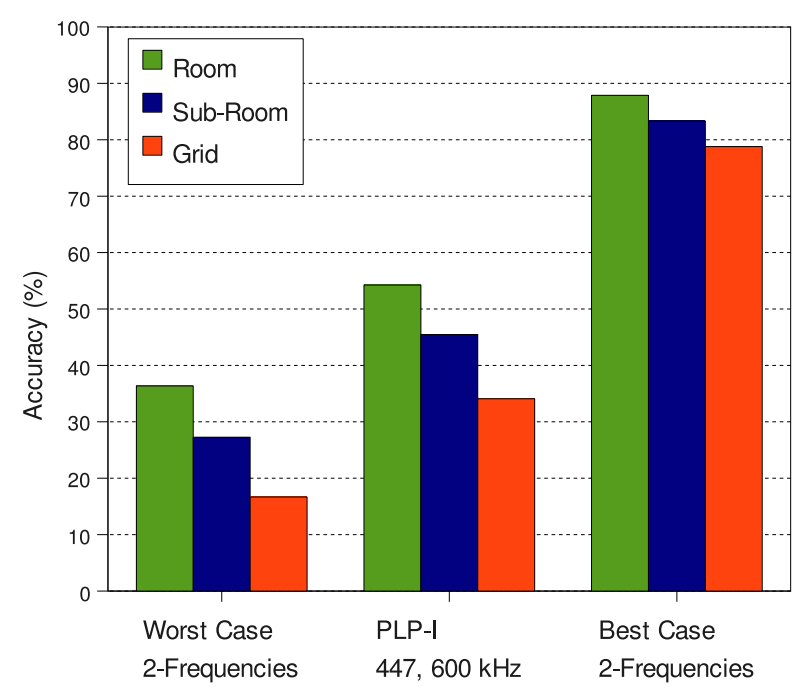

Figure 3. Localization accuracy for the two PLP-I frequencies, and also the best and worst possible accuracies with two frequencies chosen from the set of 44 that were tested. Sets of two frequencies generating the best and worst accuracies were chosen independently for each of the three localization granularities.

\section{Experimental Procedure}

When capturing data the cart was moved in succession to each point on the grid (marked with tape on the floor), where a marker placed on the cart was used to align it consistently across data captures. For each frequency, a sample was taken with the signal generator output both on and off, allowing us to capture the ambient RF noise in the environment as well as the signal we were transmitting. Samples were captured using the software radio, which digitizes the raw signal coming in off the antenna. The amplitude of the frequency of interest was calculated from this raw signal using a Fourier transform. To reduce the effect of any variation in the electronics of the software radio, we directly connected its input to the ouput of the signal generator and performed a calibration before each survey.

The signal generator was controlled over a WiFi connection (which operates well outside the frequency range of interest for these experiments), allowing us to programmatically adjust its output parameters and configure the generator to output each of the 44 frequencies in sequence. The signal generator and injector box were connected to an outlet in the kitchen, illustrated in Fig. 1. Note that since we are using a fingerprinting-based approach, it is not necessary to know anything about the design or placement of the electrical infrastructure. The only purpose of the powerline is as a conduit to spread the signal used for fingerprinting throughout the space of interest.

In this manner, a measurement of the amplitude of all 44 frequencies was taken at each of the 66 grid points. Collection of this data over the entire grid for one injection point took approximately three hours. Six complete datasets were collected over a two-month period, approximately every week.

\section{REVISITING PLP-I RESULTS}

This work began as an effort to examine the feasibility of deploying PLP-I in commercial and industrial environments, and so we began by attempting to reproduce the results of the original system [16]. Since PLP-I consisted of two injectors each transmitting a single unique frequency, we simulated it by selecting two frequencies from the larger set of 44 that were tested. To replicate the original system as closely as possible, we began by selecting the original frequencies used by PLP-I ( $447 \mathrm{kHz}$ and $600 \mathrm{kHz})$. Note that although Patel et al. utilized $33 \mathrm{kHz}$ rather than $600 \mathrm{kHz}, 33 \mathrm{kHz}$ was outside the operating range of our antenna, and discussions with the authors revealed that in subsequent experiments they had switched to $600 \mathrm{kHz}$ and obtained results similar to those previously presented.

Another difference from PLP-I is that our experiments used a single injection point, rather than two physically separated injection points. Physically separating the injectors can serve to add information useful to the classifier that may not be present if both signals are transmitted from the same location. A measure of the amount of additional information provided by using a second frequency (over using just one for classification) is the correlation between the amplitudes of the two frequencies. In our case, the correlation between $447 \mathrm{kHz}$ and $600 \mathrm{kHz}$ ranged from 0.35 to 0.42 . A correlation of 1 indicates that the second frequency provides no additional information, and a correlation of 0 means that the two frequencies share no information. Patel et al. found correlations between the two injectors ranging from 0.05 to 0.6. Our values thus fall within this range, and we therefore believe the use of a single injection point for multiple frequencies serves as a valid comparison.

To evaluate the performance of PLP-I in our test environment, we took the amplitude data at $447 \mathrm{kHz}$ and $600 \mathrm{kHz}$ captured at each of the 66 surveyed points, and used this data to perform room-level, sub-room-level, and grid-level classification of a test dataset. The classification was performed using the K-Nearest-Neighbors (KNN) method on the signal space using the received signal amplitudes. Thus, each physical location on the grid (as shown in Fig. 1) had associated with it the amplitude of the two signals $(447 \mathrm{kHz}$ and $600 \mathrm{kHz}$ ) as sensed at that parciular location, as well as the symbolic label given to that grid point. Our test and training datasets were completely independent, captured several hours apart. We used a $\mathrm{K}$ value of 1 , which was found to produce optimal results under these conditions. Room-level accuracy ranged from $48.5 \%$ to $60 \%$ depending on which of the two datasets was used for training and which was used for testing, which is significantly lower than the $78 \%-100 \%$ accuracy achieved by Patel et al. in their experiments. Subroom-level performance ranged from 39.4\%-51.5\%, again much lower than the $87 \%-95 \%$ achieved in the original work. The average of the two accuracies is shown in Fig. 3 for each localization granularity.

The lower accuracies observed can be attributed to both ambient RF noise and the construction of the electrical infrastructure. As discussed earlier, although the layout of our test 


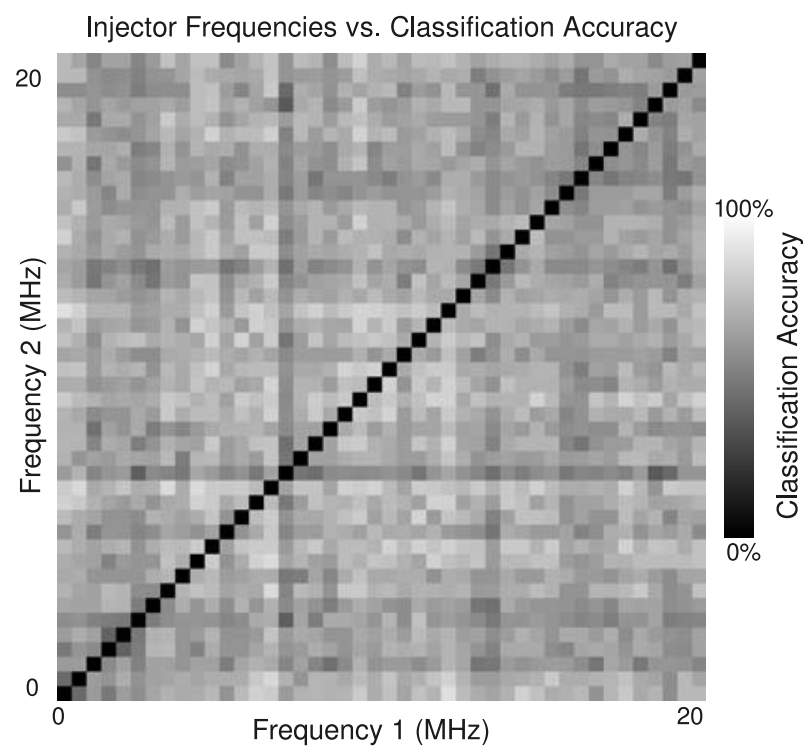

Figure 4. Room-level localization accuracy using two frequencies for each of the 1,892 possible combinations of two frequencies from the 44 that were tested. The diagonal represents no data since the injector can not transmit two signals simultaneously on the same frequency. Note the lack of any apparent pattern in the data, meaning that a formula for selecting two frequencies a priori when designing an indoor localization system is not possible.

environment appears to be that of a home, it is actually a laboratory and as such was constructed according to commercial building standards. Substantial ambient noise, possibly caused by the large amount of electrical equipment operating within the test environment, was observed in the 400$600 \mathrm{kHz}$ range. This phenomenon was not observed in the deployment of PLP-I in residential spaces. This may also be due to the electrical wiring being deployed within metal conduit, as described earlier. Note that the hot and neutral wires on which the signals are injected are completely contained within this conduit, which can serve to reduce the amount of signal radiated. The experiments conducted in PLP-I were in homes with residential grade wiring without conduit.

\section{Frequency Selection}

Given these results, we decided to perform an exhaustive search of all possible combinations of two frequencies from the 44 that were captured to determine the best accuracy achievable with two frequencies. The idea was that if a pair of frequencies could be found that consistently provide the best accuracy across each of the three classification granularities, PLP-I could be adapted to use these frequencies in commercial and industrial environments. The classification was again done using the KNN method with a $\mathrm{K}$ value of 1 .

The results of a room-level classification of each of the 66 samples taken throughout the floor are illustrated in Fig. 4 for all 1,892 possible combinations of frequencies. Lighter pixels represent better accuracy, and the diagonal of black pixels represents no data where the injector would be required to transmit the same frequency twice, which is impos-

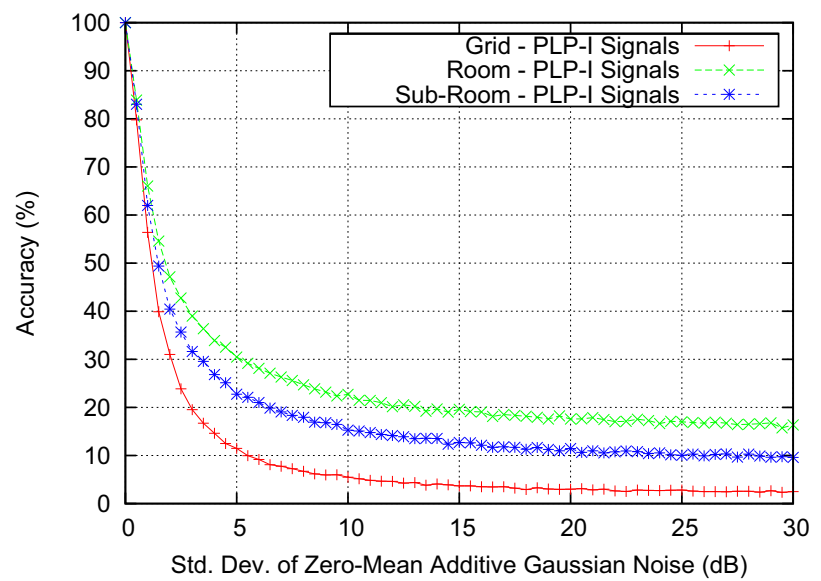

Figure 5. Localization accuracy vs. standard deviation of zero-mean additive Gaussian noise when using the two PLP-I frequencies (447 $\mathrm{kHz}, 600 \mathrm{kHz}$ ). This shows that the classifier has a high sensitivity to noise for a localization system based on the two-frequency PLP-I method.

sible. The frequency scale starts with $447 \mathrm{kHz}, 448 \mathrm{kHz}, 500$ $\mathrm{kHz}, 600 \mathrm{kHz}, 601 \mathrm{kHz}, 1 \mathrm{MHz}$, and continues in $0.5 \mathrm{MHz}$ steps to $20 \mathrm{MHz}$. The best possible room-level accuracy was $87.88 \%$ (achieved with $447 \mathrm{kHz}$ and $11.5 \mathrm{MHz}$ ), the best sub-room-level accuracy was $83.33 \%$ (achieved with 8.5 $\mathrm{MHz}$ and $11 \mathrm{MHz}$ ), and the best grid-level accuracy was $78.79 \%$ (achieved with $8.5 \mathrm{MHz}$ and $9.0 \mathrm{MHz}$ ). These results, along with those of the PLP-I frequencies and the worst-case two frequncies, are summarized in Fig. 3.

This exhaustive search leads to two important results. First, we see that significant improvements in accuracy can be made simply by using frequencies other than the $447 \mathrm{kHz}$ and 600 $\mathrm{kHz}$ signals used by PLP-I. Room-level accuracy improves by $33.6 \%$, sub-room-level accuracy by $37.9 \%$, and grid-level accuracy by $44.7 \%$ simply by using alternative frequencies. The second result of this search is that the frequencies that provide the best possible accuracy are not consistent across the three classification granularities. Not only are they inconsistent, but they also span most of the range tested, starting with the lowest frequency tested of $447 \mathrm{kHz}$ all the way to $11 \mathrm{MHz}$. Additionally, Fig. 4 fails to demonstrate any obvious pattern that would lead to a formula for a priori selection of two frequencies to be used in a PLP localization system.

\section{Temporal Stability}

For a fingerprinting-based localization system to work, the fingerprint space must exhibit both temporal stability and spatial differentiability. The previously discussed results, with room-level accuracy of up to $88 \%$ for two frequencies based on training and testing sets taken the same day, demonstrate that the amplitude of these signals is indeed spatially differentiable. We thus set out to examine both the stability of the signal amplitudes in time, as well as the effect that any instability has on localization results. 


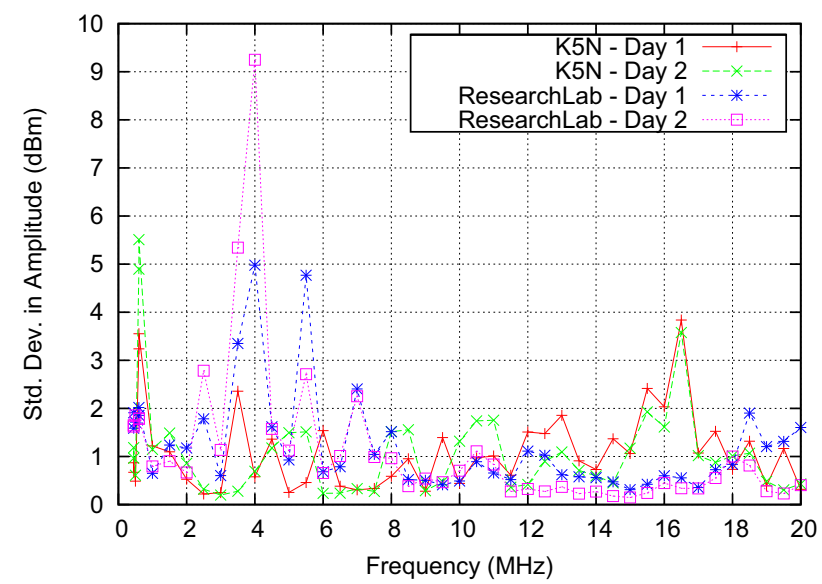

Figure 6. Standard deviation in amplitude vs. frequency for long-term tests at two physical locations. This provides an indication of the variability of the amplitude of each frequency over the course of a day. Note that numerous frequencies are above $2 \mathrm{~dB}$ in std. dev., which Fig. 5 shows will reduce localization accuracy to below $50 \%$.

To understand the impact of any temporal instability in the signal amplitudes, we ran tests for each dataset where the original dataset was used for training, and a noisy version of that same dataset was used as the subsequent testing set. The noisy version was generated by adding independently generated samples of zero-mean Gaussian noise to the amplitude of each of the PLP-I frequencies at each of the 66 physical locations in the dataset. The impact of noise with standard deviation ranging from $0 \mathrm{~dB}$ to $30 \mathrm{~dB}$ was examined, and is shown in Fig. 5. The accuracies shown represent the mean over 100 independent generations of the noisy dataset versions, as well as over all collected datasets. These results show that with just $1 \mathrm{~dB}$ of noise, the localization accuracy drops below $50 \%$ for all classification granularities when using the PLP-I frequencies of $447 \mathrm{kHz}$ and $600 \mathrm{kHz}$.

The next question then is what amount of temporal instability in the signal amplitudes exists in the real-world. To answer this, we took measurements over four separate 24hour periods at two physical locations. Approximately 950 samples at each of the 44 frequencies were obtained across each 24-hour period. One of these locations was at point K5 on the grid, and the other was in our laboratory space. During these tests the cart containing the equipment remained completely stationary, eliminating any change in signal amplitude that may be present in the datasets taken over the entire grid due to inconsistent cart placement between measurements. The results of these long-term tests are illustrated in Fig. 6, which shows the mean standard deviation in amplitude at each of the 44 frequencies across the 24 hours of each test.

Figure 6 leads to two important conclusions. First, the mean standard deviation across all four tests and all 44 frequencies is $1.17 \mathrm{~dB}$, which is above the $1 \mathrm{~dB}$ that was determined earlier to cause classifier accuracy to drop below 50\%. Three out of the four 24-hour periods had 21 frequencies with standard deviations above $1 \mathrm{~dB}$, and one had 15 frequencies

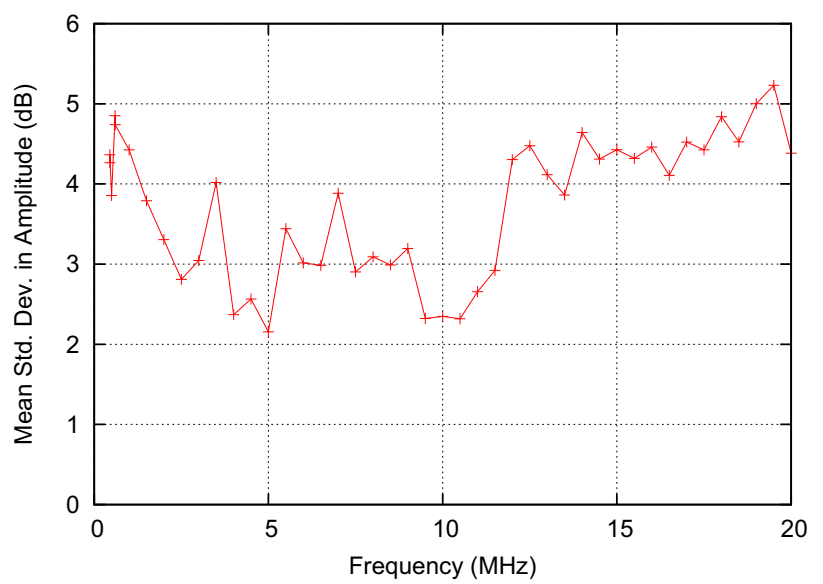

Figure 7. Standard deviation in amplitude vs. frequency. The standard deviation is found using all six independent datasets captured over the two-month period at each of the 66 surveyed points, and all points are then averaged. This gives an indication of the amount of variability in the signal amplitude that can be expected at each frequency over a more meaningful timescale than that of Fig. 6, which presented results for $\mathbf{2 4}$ hours. Unlike the stationary test though, these results can include variability due to slight inconsistencies in the placement of the receiver between dataset captures.

above $1 \mathrm{~dB}$. Of these same tests, two had five frequencies with standard deviations above $2 \mathrm{~dB}$, one had six, and one had three. Figure 5 shows that at $2 \mathrm{~dB}$ of standard deviation, localization accuracy ranges from $25 \%$ to $35 \%$ depending on localization granularity. Second, we see that although some frequencies show low standard deviation $(<1 \mathrm{~dB})$ in one location, they may experience high standard deviation $(>2 \mathrm{~dB})$ in another location. Therefore, it is impossible to select two frequencies that will produce consistently good localization results over both time and space.

A real-world deployment will obviously last much longer than the 24-hour tests we described, and will also suffer from additional 'noise' due to inconsistent placement of the receiver between training and testing samples. To get an indication of what real-world standard deviation in the signal amplitude might look like, we calculated the same value as before (standard deviation in amplitude at each of the 44 frequencies) across all six datasets. These values are shown in Fig. 7. Values were computed by finding the standard deviation among the six amplitude samples at each frequency for each of the 66 surveyed locations, and then taking the mean over all 66 locations. For the six datasets we collected over a two month period, the mean standard deviation in amplitude across all 44 frequencies was $3.74 \mathrm{~dB}$, and the minimum was $2.16 \mathrm{~dB}$. Recall that the localization accuracy of a twofrequency system is less than $35 \%$ with noise of greater than $2 \mathrm{~dB}$ (shown in Fig. 5).

\section{WIDEBAND PLP (WPLP)}

Thusfar we have demonstrated that a general rule for selection of two frequencies for PLP does not seem to exist. Additionally, even if the optimal two frequencies are selected initially, their performance may degrade over time. We thus set out to examine the effect of using additional frequencies 


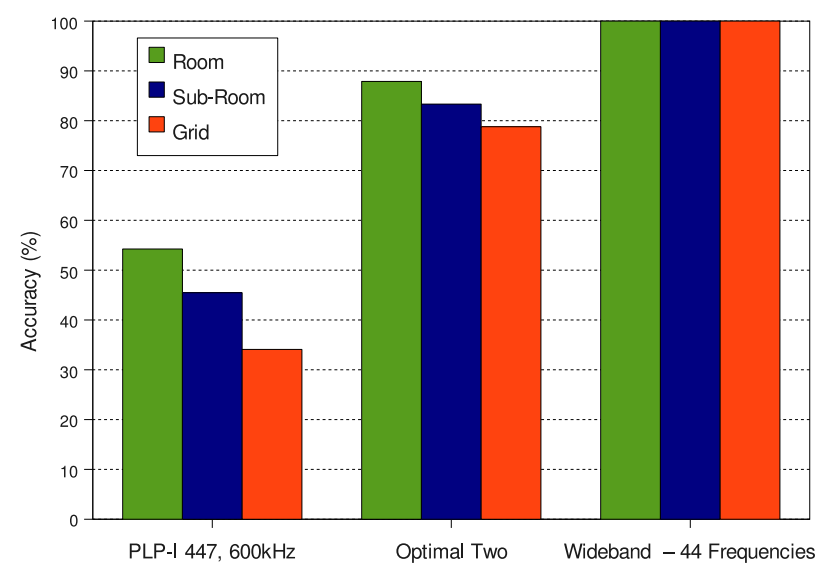

Figure 8. Localization accuracy when the classifier is trained and tested on independent datasets taken on the same day, several hours apart. Accuracy is shown for the 44-frequency wideband signal as well as the two-frequency PLP-I signal $(447 \mathrm{kHz}$ and $600 \mathrm{kHz})$ and the optimal two frequencies from all possible combinations (as shown in Fig. 3) .

in the system. In this section, we demonstrate that the use of a wideband signal consisting of signals at 44 distinct frequencies not only improves initial localization accuracy, but also better maintains that accuracy over time by mitigating some of the effects of temporal instability in each of the constituent signals.

\section{Accuracy Improvements}

The use of a 44-frequency wideband signal can significantly improve localization accuracy. Figure 8 shows localization performance for a training and testing set taken several hours apart, which should yield excellent localization results since changes in the physical environment that could affect the RF signals will be minimal. However, as we saw earlier in Fig. 3 , this is not necessarily the case when using only two frequencies for localization. In fact, the PLP-I system achieves only $54 \%$ room-level accuracy with $447 \mathrm{kHz}$ and $600 \mathrm{kHz}$, even for these relatively ideal conditions. A wideband signal provides additional features to the classifier, allowing it to make better decisions. In this case, the wideband signal leads to $100 \%$ accuracy at room, sub-room, and grid level. This represents a gain of $46 \%-66 \%$ over the accuracy achieved with the PLP-I frequencies. Figure 8 also shows that even with the optimal selection of two frequencies (with each of the three localization granularities having its optimal frequencies selected independently), the wideband signal provides gains in accuracy of $12 \%-21 \%$.

\section{Temporal Stability}

Using a wideband signal for localization not only improves initial performance of the system, but also helps maintain that performance over time. Having observed the effect that a wideband signal has on initial localization results, we set out to quantify its effect on sensitivity to noise. Noise sensitivity of the classifier was first shown in Fig. 5, which presented results for a system utilizing the two PLP-I frequencies of $447 \mathrm{kHz}$ and $600 \mathrm{kHz}$. Figure 9 illustrates this

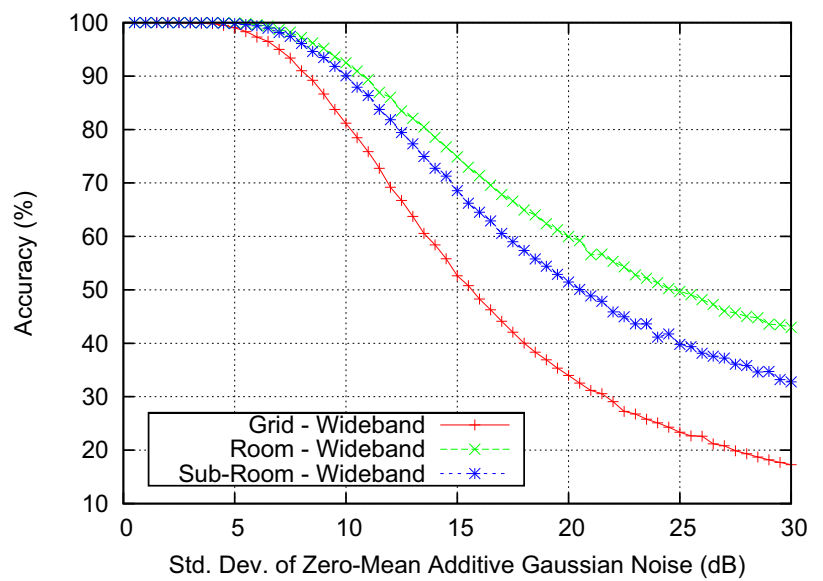

Figure 9. Localization accuracy vs. standard deviation of additive zeromean Gaussian noise. The classifier can tolerate significantly more noise in a wideband signal before accuracy degrades, and the degradation is more graceful than the two-frequency localization approach of PLP-I, shown in Fig. 5.

information for the 44-frequency wideband signal. As before, this information was obtained by adding independently generated zero-mean Gaussian noise samples with varying standard deviation to the amplitude of each of the 44 frequencies sampled at each of the 66 datapoints. The classifier was then trained on the initial dataset, and tested on the noisy version of that same dataset. Figure 9 represents the mean over 100 independent generations of a noisy version of all six datasets.

Figure 9 shows that a wideband signal is capable of tolerating noise of up to $5 \mathrm{~dB}$ in standard deviation before classifier accuracy drops below $100 \%$. In contrast, Fig. 5 demonstrates that the PLP-I two-frequency signal has accuracy of less than $25 \%$ for all localization granularities at this level of noise. In addition, Fig. 9 shows that as the noise increases, classifier accuracy degrades much more gracefully than in the PLP-I two-frequency signal.

With results showing that a wideband signal can improve temporal stability by increasing the noise tolerance of the classifier, we now discuss the results of a real-world experiment with a 44-frequency wideband signal for training and testing data taken two months apart. The results for the two-frequency PLP-I signal, the optimal two frequency signal (with optimal selection being independent for each level of localization granularity), and the 44-frequency wideband signal are shown in Fig. 10. Two important effects can be observed here, and in Fig. 11, which illustrates the decrease in performance for each of the three types of signals from initial deployment (Fig. 8) to two months later (Fig. 10). First, although a two-month temporal separation between training and testing data has reduced the accuracy of the 44frequency wideband signal to $59 \%$ - $91 \%$ depending on the localization granularity, it has maintained significantly better accuracy than either the two-frequency PLP-I signal or the optimal two-frequency signals across all three localization granularities. Second, the decrease in performance with the 


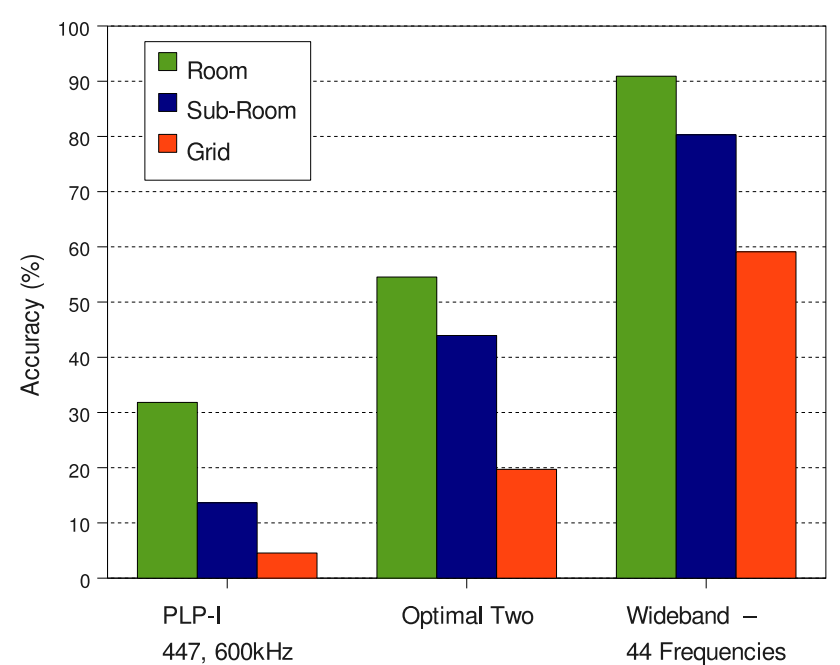

Figure 10. Localization accuracy when the classifier is trained and tested on independent datasets taken two months apart. Accuracy is shown for the two-frequency PLP-I signal $(447 \mathrm{kHz}$ and $600 \mathrm{kHz})$, the optimal two frequencies from all possible combinations, and the 44frequency wideband signal.

44-frequency wideband signal for all localization granularites (except grid-level) is lower than with the two-frequency PLP-I signal and the two-frequency optimal signal. Thus, not only does a wideband signal deliver better initial accuracy, it also serves to better maintain that accuracy over time.

The data discussed thus far is based on the comparison of three independently collected datasets - two separated by several hours, and one separated from the initial set by two months. The earliest dataset has been used as the training set, which simulates the initial site survey necessary upon deployment of a fingerprinting-based localization system. Over the two-month period, we collected three additional datasets in the interim. Using these interim datasets as additional testing data for a classifier trained on the initial site-survey can provide insight into how localization accuracy degrades over time, and is shown in Fig. 12. The interesting result here is that there does not appear to be a pattern in accuracy degradation over the two-month period as time increases. It appears that any significant temporal separation between training and testing datasets will degrade accuracy, but that accuracy degradation is not necessarily correlated with increasing time after a point.

\section{Wideband Signal Size}

Evidence clearly shows the benefit of the wideband signal over a two-frequency signal, although it is unclear if a wideband signal with fewer than 44 constituent frequencies may provide the same accuracy. Using the fewest number of signals possible to obtain the demonstrated benefits of a wideband signal is desirable since additional frequencies may increase the cost, complexity, and size of receivers designed for a real-world practical localization system based on the wideband approach.

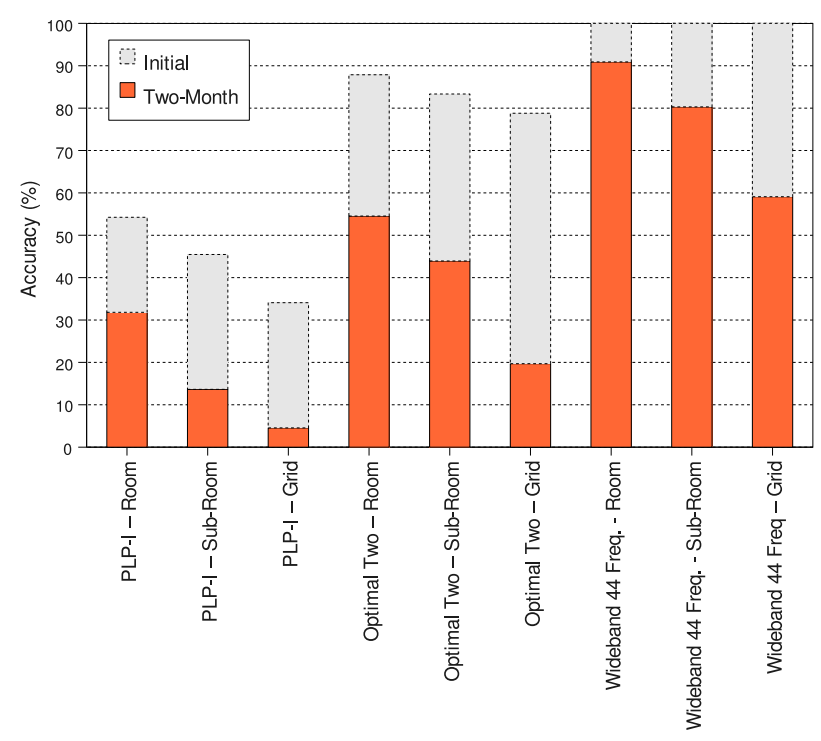

Figure 11. Classifier performance degradation between closely temporally spaced training and testing data (several hours), and a two-month separation between training and testing data. Red bars represent the localization accuracy after two-months, and the combined red and gray bars represent the initial localization accuracy soon after deployment (the closely spaced training and testing data). A 44-frequency wideband signal shows both better initial accuracy and reduced accuracy degradation over time.

To determine the effect of additional frequencies on localization performance, we examined the performance of a wideband signal ranging in size from five frequencies all the way to the complete set of 44 frequencies on the two-month separated datasets. Since a key benefit of the use of a wideband signal is resistance to performance degradation over time, examining the two-month separated data makes more sense than the datasets captured closer together in time. To determine the order in which frequencies were added to the signal, they were ranked in order of decreasing signal-tonoise ratio (SNR) based upon the training dataset. Although other orderings could be used, and may produce different results, the problem space is too large to perform an exhaustive analysis. Adding frequencies by decreasing SNR is a logical approach since frequencies closer to the ambient noise level are more likely to be affected by noise, and will thus have a higher standard deviation in amplitude. Recall Figs. 5 and 9, which showed that this adversely affects localization performance. Frequencies with higher SNR's will thus likely provide better localization accuracy. Localization accuracies for the wideband signal ranging in size from five to 44 frequencies are shown in Fig. 13. The general trend is that the use of additional frequencies provides additional localization accuracy, although it appears that the marginal utility of each additional frequency begins to decrease past 35 frequencies.

\section{DESIGNING A DEPLOYABLE SYSTEM}

While our initial experimental platform consists of a relatively complex and expensive set of test equipment, the flexibility of this equipment is desirable for the testing and rapid 


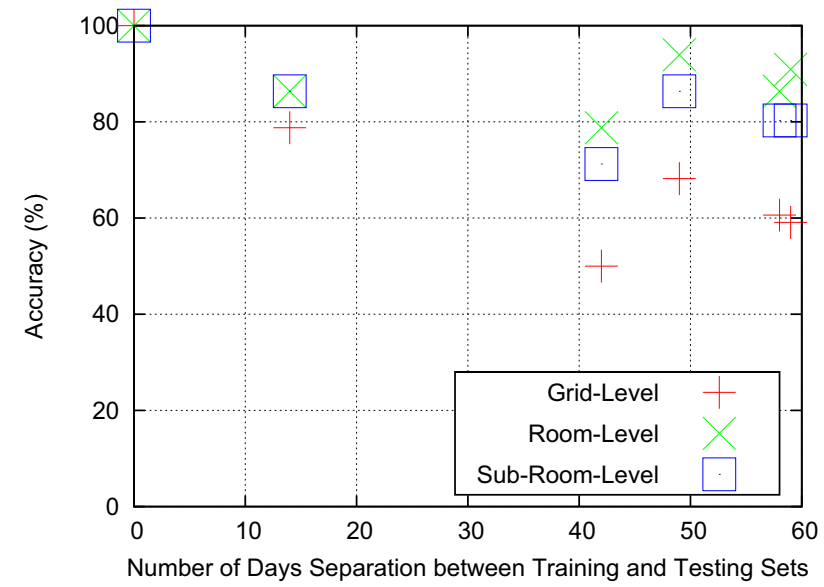

Figure 12. Localization accuracy vs. number of days separation between the training and testing data for a 44-frequency wideband signal. Accuracy does not seem to continually decrease over the two-month period, although an initial decrease in accuracy with any time separation between training and testing data is apparent.

prototyping of new configurations of PLP variants. Once we have established the feasibility of a specific system configuration using the test apparatus, we can then design special purpose hardware and software that is optimized for a specific set of operating parameters, including the desired operating frequency range, injector transmission levels, and receiver dynamic range and selectivity.

Interference with other wireless devices is a potential concern with WPLP. Wired power line communication systems operate within the frequency band of interest for WPLP, as well as various other wireless services such as amateur radio. Since WPLP uses a number of frequencies, the system could overcome potential interference issues by continually sensing for other users of the various frequencies and adapting its frequency set in real-time. This method has been used successfuly with power line communication systems, which emit unintentional radiation from the power lines.

A candidate design could leverage several technologies to achieve good wideband performance at modest cost and complexity, as well as low power consumption for the PLP receiver. Having demonstrated the value of a 44 frequency wideband signal, we now present a possible design for a practical wideband-capable receiver.

\section{Injector: Single Chip Direct Digital Synthesis}

In our experimental apparatus, the injector system employs an Agilent 33220A signal generator, which is based on an arbitrary waveform generator architecture where a time series of arbitrary output voltages is played back from a large internal memory. In our implementation, however, we are not using the full arbitrary waveform generation capabilities of this generator as the injector transmits only 1 of 44 frequencies at a time. In a practically deployable implementation of the wideband solution, a single chip direct digital synthesizer could be employed to generate frequency agile single excitation tones. The Analog Devices AD9834, for

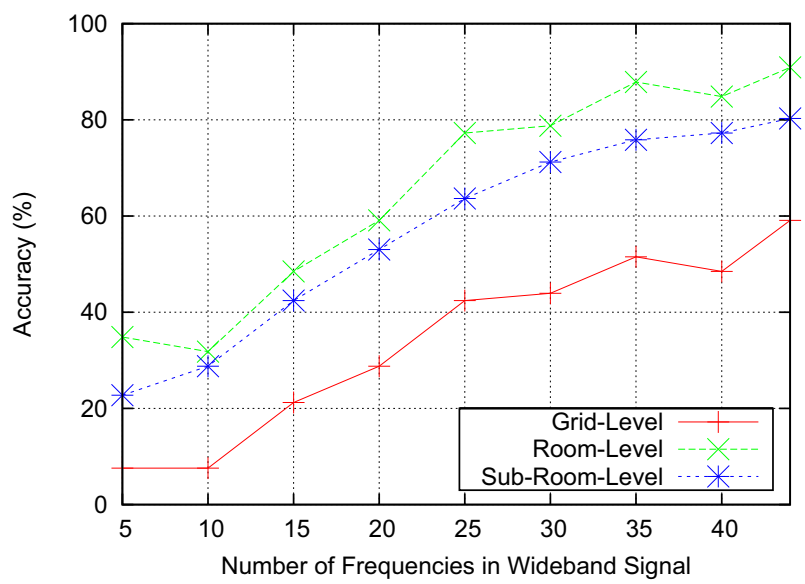

Figure 13. Localization accuracy vs. number of frequencies in the wideband signal when trained and tested on data separated by two months. The use of additional frequencies provides additional features for the classifier and generally improves accuracy. Frequencies were added from the set of 44 in order of decreasing signal-to-noise ratio (SNR).

example, is capable of generating tones at frequencies ranging from near-DC to $37.5 \mathrm{MHz}$. This IC may be controlled by a simple 8 -bit microcontroller programmed to generate a sequence of injection tones as required. A high-speed operational amplifier would be required to amplify the AD9834's $0.8 \mathrm{~V}$ output signal to the $10 \mathrm{~V}$ output signal we employed in our tests.

\section{Receiver: Single Chip Superheterodyne Receiver}

In our experimental apparatus, the receiver system employs a large broadband receiving loop antenna connected to a software radio development system (USRP) which is in turn connected to an ordinary laptop computer which performs some of the signal processing tasks required. This combination of components was chosen for flexibility and high performance, but it consumes a great deal of power and is physically very large. In a practical wideband receiver, a single chip superheterodyne receiver such as the NXP (formerly Philips) TEA5757 contains on a single chip all of the receiver functions needed for measuring the amplitude of incoming signals from $144 \mathrm{kHz}$ to $30 \mathrm{MHz}$. In operation, this IC consumes $18 \mathrm{~mA}$ at $2.5 \mathrm{~V}$, or $45 \mathrm{~mW}$, which is about half the power consumption of a typical Bluetooth transceiver implementation. A microcontroller would be required to configure the receiver IC to various frequencies. The real challenge for the production of a small WPLP tag is the development of a small but sensitive magnetic field antenna to receive the incident signals from the power line without requiring a large shielded loop. Initial tests show that an untuned ferrite rod antenna holds promise for this application.

\section{FUTURE WORK}

Although we believe the work presented here represents a significant result in the space of fingerprinting-based indoor localization, many opportunities exist for future work. First, this work began as an attempt to make PLP-I operate in noisy 
commercial and industrial spaces. While we have achieved that goal in the tested space, performance of WPLP needs to be examined in other spaces. Second, although Fig. 13 seems to demonstrate diminishing returns for further expansion of the wideband signal beyond 44 frequencies, the data is by no means conclusive and we believe it would be beneficial to examine larger wideband signals, as well as wideband signals using other areas of the spectrum, such as the $\mathrm{GHz}$ region used for WiFi localization. Finally, we believe this work presents opportunities for investigation of alternative machine learning techniques, particularly support vector machines.

\section{CONCLUSION}

In this work we have shown that fingerprinting-based localization systems are susceptible to accuracy degradation over time due to variability in the fingerprint. For a system such as PLP whose fingerprint is the amplitude of a limited number of RF signals, this variability comes in the form of ambient RF noise as well as changes over time in the transmitting and receiving hardware. We then showed that the use of a wideband signal consisting of the amplitudes of 44 different frequencies ranging from $447 \mathrm{kHz}$ to $20 \mathrm{MHz}$ can both improve the initial post-deployment accuracy of the system, and also mitigate some performance degradation over time. We also show that, at least up to the maximum of 44 frequencies tested, increasing the size of the wideband signal fingerprint serves to reduce accuracy degradation over time.

Although our testing apparatus is clearly not designed with a deployable system in mind due to its large size, this does not make the results presented here uninteresting. A real-world system which takes advantage of the wideband concept can easily be designed with off-the-shelf components in the future.

\section{ACKNOWLEDGMENTS}

The authors wish to thank Yi Han for assistance in data collection and development of data visualization tools, as well as the entire UbiComp Research Group for their feedback throughout the writing of this paper.

\section{REFERENCES}

1. Wellbrook communications ala-1530+ antenna. http://www.wellbrook.uk.com/products.html.

2. Indoor gps. http://www.indoorgps.com (2007).

3. Vicon mx, http://www.vicon.com/products/systems.html.

4. BAhl, P., AND PAdMAnabhan, V. Radar: An in-building rf-based user location and tracking system. In IEEE Infocom (2000), pp. 775-784.

5. EKAHAU. http://www.ekahau.com.

6. Global Search Communications, I. http://www.globalsearch.ws.

7. HAZAS, M., AND WARD, A. A novel broadband ultrasonic location system. In Ubicomp 2002 (2002).

8. HAZAS, M., AND WARD, A. Broadband ultrasonic location systems for improved indoor positioning. In IEEE Transactions on Mobile Computing (2006).

9. Hightower, J., AND Borriello, G. A survey and taxonomy of location systems for ubiquitous computing. Tech. Rep. CSC-01-08-03, University of Washington, 2001.
10. KJRGAARD, M. Towards a taxonomy for location fingerprinting. In $L o C A$ (2007).

11. Krumm, J., Cermak, G., , AND Horvitz, E. Rightspot: A novel sense of location for a smart personal object. In Ubicomp (2003).

12. LAASONEN, K. Clustering and prediction of mobile user routes from cellular data. In $P K D D$ (2005).

13. Lamarca, A., Chawathe, Y., Consolvo, S., Hightower, J., Smith, I., Scott, I., Sohn, T., Howard, J., Hughes, J., Potter, F., TABert, J., Powledge, R., Borriello, G., , AND Schilit, B. Place lab: Device positioning using radio beacons in the wild. In Pervasive 2005 (2005).

14. Madhavapeddy, A., AND Tse, T. Study of bluetooth propagation using accurate indoor location mapping. In Ubicomp 2005 (2005).

15. Otsason, V., Varshavsky, A., A., L., And de Lara, E. Accurate gsm indoor localization. In Ubicomp 2005 (2005).

16. Patel, S., Truong, K., And Abowd, G. Powerline positioning: A practical sub-room-level indoor location system for domestic use. In Ubicomp 2006 (2006).

17. Priyantha, N. B., Chakraborty, A., And BALAKRISHNAN, H. The cricket location-support system. In Mobile Computing and Networking (Mobicom 2000) (2000).

18. Rekimoto, J., AND AyatsukA, Y. Cybercode: Designing augmented reality environments with visual tags. In In the proceedings of Designing Augmented Reality Environments (DARE 2000) (2000).

19. Rekimoto, J., AND Katashi, N. The world through the computer: Computer augmented inter-action with real world environments. In UIST 1995 (1995).

20. UBISENSE. http://www.ubisense.net.

21. Want, R., Hopper, A., Falcao, V., , And GibBons, J. The active badge location system. In ACM Transactions on Information Systems (1992).

22. Ward, A., Jones, A., AND Hopper, A. A new location technique for the active office. IEEE Personal Communications 4, 5 (1997), 42-47. 